\section{Jugendvorsorge für Flüchtlinge}

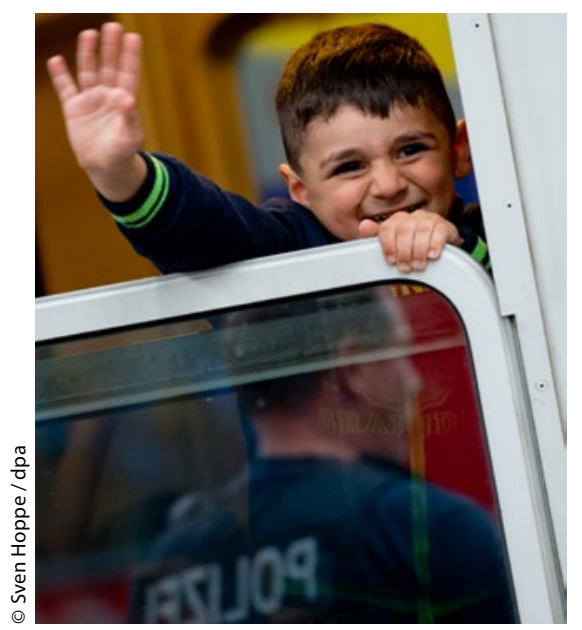

— Der Strom der Flüchtlinge reißt nicht ab. Dabei rückt die medizinische Versorgung insbesondere von minderjährigen Flüchtlingen zunehmend in den Fokus. Kinder- und Jugendarzt Dr. Thomas Nowotny forderte beim diesjährigen Kinder- und Jugendärztekongress der vier großen pädiatrischen Fachgesellschaften die Einführung einer

\section{GBE kompakt Angehörige im Fokus}

— Etwa 4,7 Millionen Menschen - 7\% der erwachsenen Deutschen - kümmern sich regelmäßig um eine pflegebedürftige Person. 65\% der Pflegenden sind Frauen, 35\% Männer. Das Robert Koch-Institut hat mit dem neuen GBE (Gesundheitsberichterstattung) kompakt "Pflegende Angehörige Deutschlands größter Pflegedienst" aktuelle bundesweit repräsentative Daten zur Situation pflegender Angehörigen vorgelegt. Ein Drittel der Pflegenden erbringt danach täglich mindestens zwei Stunden Pflegeleistungen (Pflegende mit hohem Pflegeumfang). Diese Angehörigen schätzen im Vergleich zu Nicht-Pflegenden ihren Gesundheitszustand häufiger als nicht gut ein. Zum Beispiel ist bei pflegenden Frauen die Wahrscheinlichkeit höher, keinen Sport zu treiben, zu rauchen und keine Zahnvorsorge in Anspruch zu nehmen.

www.rki.de
Jugendvorsorge für unbegleitete minderjährige Flüchtlinge (J-umF). Als Basis könnten die Jugendgesundheitsuntersuchungen $\mathrm{J} 1$ und $\mathrm{J} 2$ dienen, die aber stark modifiziert werden müssten. So sollten zum einen die Altersgrenzen aufgehoben werden, zum anderen sei es erforderlich, die Untersuchungen inhaltlich zu erweitern. Neben der Erhebung des Impfstatus sowie der Anamnese von Infektionskrankheiten und einer Vitamin-D-Prophylaxe fordert der Pädiater, den Fokus auch auf mögliche psychische Probleme zu legen. Der Einsatz eines bereits entwickelten und auf die speziellen Bedarfe der Flüchtlingskinder ausgerichteten Fragebogens soll helfen, seelische Verletzungen und traumatische Erlebnisse zu ermitteln. Dabei sollte unter anderem nach den Ereignissen auf der Flucht, den Ängsten und Albträumen und verbreiteten Folgewirkungen sowie nach Kopfschmerzen, Schlafstörungen und Posttraumatischen Belastungsstörungen (PTBS) gefragt werden.

Nach Informationen der Ärzte Zeitung

\section{Hier steht eine Anzeige.}

\author{
Springer
}

\section{DRK}

\section{Pflegekräfte entlasten}

- Das Bundeskabinett wird im Oktober über die Krankenhaus-Reform abstimmen. Das Deutsche Rote Kreuz und der Verband der Schwesternschaften vom DRK e.V. erkennen die Fortschritte an, haben jedoch Befürchtungen. „Das Gesetz bietet aus unserer Sicht keine dauerhaften Lösungen bzw. Antworten auf Zukunftsfragen und Personalengpässe - vor allem in der Pflege", betont die Präsidentin des Verbandes der Schwesternschaften vom DRK e.V., Generaloberin Gabriele Müller-Stutzer. Seit 1995 seien ca. 50.000 Vollzeitstellen an deutschen Kliniken abgebaut worden. Bis 2030 werde es in Deutschland drei Millionen Behandlungsfälle mehr geben. „Bei der skizzierten Entwicklung wird die ohnehin schon hohe Arbeitsbelastung der Pflegekräfte in den Kliniken aufgrund des Personalmangels weiter steigen", befürchtet Müller-Stutzer.

www.rotkreuzschwestern.de 